\title{
Primary Human Monocytes Differentiate into M2 Macrophages and Involve Notch-1 Pathway
}

\begin{tabular}{|r|l|}
\hline Journal: & Canadian Journal of Physiology and Pharmacology \\
\hline Manuscript ID & cjpp-2016-0319.R1 \\
\hline Manuscript Type: & Article \\
\hline Date Submitted by the Author: & 21 -Oct-2016 \\
\hline Complete List of Authors: & $\begin{array}{l}\text { Singla, Dinender Kumar; University of Central Florida, } \\
\text { Wang, Jing; University of Central Florida, College of Medicine } \\
\text { Singla, Reetu; University of Central Florida, College of Medicine }\end{array}$ \\
\hline Keyword: & monocyte, macrophage, Notch-1, atherosclerosis, DAPT, siRNA \\
\hline \multicolumn{2}{|l}{} \\
\hline
\end{tabular}




\title{
Primary Human Monocytes Differentiate into M2 Macrophages and Involve Notch-1 Pathway
}

\author{
Dinender K. Singla, Jing Wang, Reetu Singla
}

Division of Metabolic and Cardiovascular Sciences, Burnett School of Biomedical Sciences, College of Medicine, University of Central Florida, Orlando, FL 32816, USA

Short Title: Human Monocyte Differentiation into M2 Macrophages

\section{Address for Correspondence:}

Dinender K. Singla, PhD, FAHA, FIACS

Division of Metabolic and Cardiovascular Sciences,

Burnett School of Biomedical Sciences, College of Medicine

University of Central Florida,

4110 Libra Dr., Room 321

Orlando, FL, 32816, USA

Email: dsingla@ucf.edu

Phone: 407-823-0953

Fax: $\quad 407-823-0956$ 


\section{Abstract}

The current study investigates whether inhibiting the Notch-1 signaling pathway in primary human monocytes enhances M2 macrophage differentiation. We generated primary human monocytes cell culture model to understand the effect of Notch-1 signaling pathway. Monocytes were treated with Notch-1inhibitors DAPT or siRNA. Our data show that there was a significant increase in the M1 macrophage population demonstrated by iNOS marker in the primary human monocytes treated with apoptotic conditioned medium (ACM). Next, the levels of pro-inflammatory cytokines IL-6, and MCP-1, as well as TNF- $\alpha$ increased in ACM media $(p<0.05)$. Furthermore, M1 macrophages and pro-inflammatory cytokines were reduced following DAPT or siRNA treatment. Comparatively, there was a significant increase in M2 macrophages as demonstrated by an increase in CD206 and arginase-1 positive cells treated with DAPT or siRNA $(p<$ 0.05). Furthermore, a significant increase in the associated anti-inflammatory cytokines IL-10 and IL-1RA was also observed with respect to control groups $(p<$ 0.05). We conclude that blocking the Notch-1 pathway with DAPT or siRNA attenuates pro-inflammatory cytokines, enhances M2 macrophage differentiation, and increases anti-inflammatory cytokines in primary human monocytes. As a result, Notch-1 pathway inhibition has potential therapeutic applications of inflammatory disease.

Key Words: monocyte, macrophage, Notch-1, atherosclerosis, DAPT, siRNA 


\section{Introduction}

Atherosclerosis (ATH), characterized by plaque accumulation in the arteries, is notably dangerous to cardiovascular health (Fukuda and Aikawa 2013; Kleeman et al. 2011; Moore and Tabas 2011; Moore et al. 2013). Of distinct interest are macrophages, as they are key responders in inflammatory processes (Fung et al. 2007; Moore et al. 2013; Outtz et al. 2010). When an injury occurs, macrophages migrate to the area and differentiate into two polarized subclasses, M1 and M2 macrophages (Kigerl et al. 2009; Rocher and Singla 2013). Understanding the mechanisms by which these macrophages polarize and their specific roles in disease states are key to identifying novel treatments for ATH.

Both subclasses of macrophages, M1 and M2, differentiate from a broad class of cells called monocytes (Daigneault et al. 2010). THP-1 cells, a monocyte cell line, have been used extensively in the investigation of macrophage polarization and differentiation (Qin 2012). Theoretically, THP-1 cells are morphologically and functionally similar to primary monocytic cells, and are believed to mimic the role of primary monocytes (Qin 2012). In the context of ATH and other inflammatory diseases, primary human monocytes, obtained directly from patient or healthy controls, are used to understand the functional significance of their mechanistic pathways by generating cell culture and in vivo models.

Various environmental stimuli in cell culture or in vivo determine which subclass of macrophage the monocyte differentiates. M1 macrophages have 
been found to be activated by IFN- $\gamma$, TNF- $\alpha$, and LPS (found in certain bacteria), and are pro-inflammatory (Schildberger et al. 2013; Wolfs et al. 2011). M2 macrophages are activated by IL-4 and IL-13, and are anti-inflammatory (Wolfs et al. 2011).

The pro-inflammatory cytokines secreted by M1 macrophages are traditionally thought of as a defense that the organism produces when infected by a foreign object or organism (Wolfs et al. 2011). While they are helpful in the previous case, they are detrimental in certain disease pathologies, such as ATH, by releasing pro-inflammatory cytokines, and therefore furthering tissue damage (Aoyama et al. 2009; Wolfs et al. 2011). These pro-inflammatory cytokines include IL-6, MCP-1, and TNF- a (Kleemann et al. 2008; Outtz et al. 2010; Singla et al. 2014). Conversely, M2 macrophages are anti-inflammatory, and aid in the repair of damaged tissue, making them a potential candidate for therapeutic options (Rocher et al. 2012; Singla et al. 2016). Secreted from M2 macrophages are the anti-inflammatory cytokines IL-10 and IL-1RA, and show an increase in the expression of Arginase-1 enzyme important in reducing harmful nitric oxide production (Kleemann et al. 2008; Outtz et al. 2010; Singla et al. 2014).

The exact role of macrophage subtypes in inflammatory diseases and their mechanisms of differentiation are still unknown. We recently reported a cell culture model using THP-1 cells that the inhibition of the Notch-1 pathway enhances monocyte differentiation into M2 macrophages (Singla et al. 2014). The blocking of this pathway has been achieved in previous studies by the 
gamma secretase inhibitor (GSI) known as DAPT, and small interfering RNA (siRNA) to knock down the function of the Notch-1 receptor (Singla et al. 2014).

The significance of this paper is to understand whether primary human monocytes can differentiate into M2 macrophages by blocking the Notch-1 pathway. We used DAPT to block the Notch-1 pathway, which is considered to be non-specific, we then used the specific inhibitor, Notch-1 receptor siRNA, to block pathway activation. This purpose of this study was to determine if inhibiting the Notch-1 pathway in primary human monocytes will decrease the presence of M1 macrophages and their cytokines, and subsequently enhance M2 macrophage differentiation and promote release of anti-inflammatory cytokines. 


\section{Materials and Methods}

\section{Primary Monocyte Cell Culture}

The primary human monocytes were obtained from Sanguine Biosciences (Human PBMC-005a, Bio Supply Net, New York). The frozen samples were thawed as instructed by the manufacturer and were directly placed in a $37^{\circ} \mathrm{C}$ water bath, and agitated until thawed. The vial was cleaned as directed, and then within 30 seconds, $1 \mathrm{ml}$ of medium was slowly added to the cells, then followed by an additional $8 \mathrm{ml}$ of the media. The tube was inverted and pipetted up and down to mix. The vials were then centrifuged for 10 minutes at $400 \mathrm{xg}$. The supernatant was removed, and the pellet was resuspended in $10 \mathrm{ml}$ of media. Cells were placed in the incubator at $37^{\circ} \mathrm{C}$. Cells were maintained using standard cell culture medium.

\section{Cell Culture Model}

Apoptotic-conditioned media (ACM) was used in order to simulate cellular stress on primary human monocyte cells. This was used as previously reported in THP-1 cell culture studies by us (Singla et al. 2014). To summarize the main points of the protocol, $\mathrm{H} 9 \mathrm{c} 2$ cardiomyoblasts were plated for $24 \mathrm{~h}$ in $60-\mathrm{mm}$ tissue culture dishes with $5 \times 10^{5}$ cells in each plate. 24 hours after that, $400 \mu \mathrm{M}$ of $\mathrm{H} 2 \mathrm{O} 2$ was added to each plate for 2 hours, and then the ACM was removed and filtered.

DAPT, a GSI, was also used to inhibit the functionality of the Notch-1 receptor. Protocol for DAPT treatment followed what has been previously reported by us (Singla et al. 2014). In short, the human primary monocytes were 
plated in 48-well plates, each containing 20,000 cells, and stayed there for 24 hours. 48 hours after the treatment, the cells were analyzed via immunocytochemistry. The groups were divided into control (primary human monocytes and RPMI media), ACM (primary human monocytes, RPMI media and $A C M$ ), and ACM+DAPT (primary human monocytes, RPMI media, ACM and DAPT).

Small interfering siRNA is one method used to inhibit the Notch-1 pathway. The siRNA was used per manufacturing instructions, and as previously reported (Singla et al. 2014). The groups were divided into control (scramble RNA+ primary human monocytes+RPMI), scr+ACM (scramble RNA, primary human monocytes, RPMI media and ACM), and siRNA+ACM (primary human monocytes, ACM, RPMI and siRNA).

\section{Immunocytochemistry}

In order to study the Notch-1 receptor and M1 and M2 macrophage markers, immunocytochemistry was used. The protocol previously reported by us was used (Singla et al. 2014). In short, the cell smears were prepared on ColorFrost slides, fixed with $4 \%$ paraformaldehyde, blocked for 1 hour at room temperature with $10 \%$ normal goat serum, and the primary antibodies anti-Notch1 to identify the Notch-1 receptors, anti-iNOS to identify M1 macrophages, antiCD206 to identify M2 macrophages were added. The slides were counterstained with DAPI, and then were then incubated at $4^{\circ} \mathrm{C}$ for 24 hours. Cells were photomicrographed using confocal microscopy and cell counting was preformed, as we reported previously (Rocher et al. 2012; Singla et al. 2014). 


\section{ELISA Analysis of Cytokines}

The cytokines secreted by the M1 and M2 macrophages were quantified using ELISA kits, following the manufacturer's instructions as we published (Singla et al. 2014). The kits included IL-6 (No. ELH-IL6), IL-10 (No. ELH-IL10), IL-1RA (No. ELH-IL1RA), TNF-a (No. ELH-TNFa), and MCP-1 (No. ELH-MCP1) and were purchased from RayBiotech (Norcross, GA). Developed color intensity was measured at $450 \mathrm{~nm}$ using a BioRad reader, and the obtained OD was expressed in arbitrary units (A. U.)

\section{Statistical Analysis}

Data is expressed as the mean $\pm \mathrm{SE}$, and the statistical significance was examined when $p<0.05$ using one-way ANOVA and Tukey's test. 


\section{Results}

\section{Effects of DAPT Treatment on Notch-1 Receptor and Macrophage Differentiation}

To identify M1 macrophages and Notch-1 receptors from primary human monocytes, we performed double immunostaining. Figure 1, A shows the immunohistochemical stained slides with anti-Notch-1 to identify the Notch-1 receptor(a, f, k), anti-inducible nitric oxide synthase (iNOS) to identify M1 macrophages (b, g, I), 4',6-diamidino-2-phenylindole (DAPI) to identify cell nuclei (c, h, m), the merged photomicrographs are shown in $d, i, n$, and enhanced versions of the merged images for better visualization $\mathrm{e}, \mathrm{j}, \mathrm{o}$.

Next, to determine the effect of ACM and siRNA on M2 macrophages and Notch-1 receptors, the cells were stained. Figure 1, B shows that the cells were stained with anti-Notch-1 (a, f, k), anti-CD206 to identify M2 macrophages (b, g, I), 4',6-diamidino-2-phenylindole (DAPI) (c, h, m), the merged photomicrographs are shown in d, i, n, and enhanced versions are seen in e, j, o.

We performed quantitative analysis of the Notch-1 receptor and of both M1 and M2 macrophages, in addition to the staining, in order to study the differentiation from the primary human monocytes, and the presence of receptors and cytokines both in the control and experimental groups. The data suggests that as ACM is added, the percentage of Notch-1 receptors on the M1 macrophages is significantly increased compared to the control, however, when DAPT was added, there was a significant decrease in the percentage of Notch-1 receptors found on M1 macrophages compared to the ACM experimental group 
$(p<0.05$, Fig. 1, C). The percent of M1 macrophages were significantly increased following ACM treatment compared to controls $(p<0.05$, Fig. 1, D). This increase of M1 macrophage presence was reduced post-DAPT treatment.

On the other hand, when observing the data for the percentage of Notch-1 receptors present on M2 macrophages, there was no significant alteration between the control, ACM, and ACM+DAPT groups (Fig. 1, E). When observing the M2 macrophages, it can be seen that there was a significant decrease in number when treated with ACM compared to the control, however, when DAPT was added, there was a significant increase in M2 macrophages compared to both the control as well as the ACM groups $(p<0.05$, Fig. 1, F). This suggests that DAPT treatment successfully inhibited the Notch-1 pathway, and enhanced M2 macrophage polarization.

\section{DAPT and its Effect on Inflammatory Cytokines}

As described above, IL-6, and MCP-1 are pro-inflammatory cytokines, shown in Fig. 2, A and B, respectively. IL-10 and IL-1RA are anti-inflammatory cytokines, arginase-1 is an M2 macrophage marker, and these are represented in Fig. $2 \mathrm{C}, \mathrm{D}$ and $\mathrm{E}$, respectively. These cytokines allow for determination of which type of macrophage is present, and how it has been affected by the DAPT treatment. The cytokines allow for conclusions to be drawn about whether there was successful differentiation into one of the types of macrophages, and also points to what kind of effect that they may have on disease progression or healing. 
It was observed that when primary human monocytes were placed in ACM, there was a significant increase of IL-6 and MCP-1 as compared to the control ( $p<0.05$, Fig. 2, A and B). When DAPT was added, we observed a decrease in IL-6 levels, but the values were not statistically significant (Fig. 2, A). Moreover, there was a significant decrease in MCP-1 as compared to the ACM group in B $(p<0.05$, Fig. $2, B)$.

With respect to the anti-inflammatory cytokines, it was observed that there were no significant changes to cytokine levels when placed in ACM for IL-10, IL1RA and arginase-1 (Fig. 2, C, D and E). Conversely, when DAPT was added, there was a significant increase in IL-10, and IL-1RA anti-inflammatory cytokines and arginase-1 marker observed compared to both the control and ACM groups $(p<0.05$, Fig. 2, C, D, and E). This data suggests that DAPT treatment can block the Notch-1 pathway, enhance M2 macrophage polarization and increase their released cytokines.

Effect of siRNA Treatment on Notch-1 Receptor and Macrophage

\section{Differentiation}

Since DAPT is a non-specific inhibitor for the Notch-1 receptor, further studies were performed using siRNA specific to the Notch-1R. We examined the effect of siRNA treatment on M1 and M2 macrophages through immunostaining. The photomicrographs of the macrophages are stained for anti-Notch-1, M1 and M2 macrophages. Figure 1 shows explanation of this staining.

Quantitative analysis on the receptors and cytokines was completed to determine siRNA's effect on monocytes, and their differentiation into 
macrophages. The data suggests that when primary human monocytes are treated with ACM media there is significant upregulation of the Notch-1 receptor on the M1 macrophage compared to the control ( $p<0.05$, Fig. $3, \mathrm{C})$. This increase was reduced when siRNA was added, as the percentage of the receptors on the M1 macrophages significantly decreased compared to the scr+ACM experimental group ( $p<0.05$, Fig. 3, C). The Notch-1 receptor on the M2 macrophages showed no significant alteration between the control, ACM and siRNA+ACM groups (Fig. 3, E).

Our quantitative data shows there was a significant increase in M1 macrophages, as expected under conditions of stress; however when siRNA was added, there was a significant decrease in M1 macrophages $(p<0.05$, Fig. $3, \mathrm{D})$. In contrast, when M2 macrophages were quantified, there was no significant alteration between the scr+ACM and control group; however, there was a significant increase in M2 macrophages when cells were treated with siRNA compared to both the control and the scr+ACM groups (Fig. 3, F).

\section{siRNA and its Effect on Inflammatory Cytokines}

Figure 4 shows there was a significant increase in pro-inflammatory cytokines IL-6 and MCP-1 in the scr+ACM group compared to the control, however, a significant decrease was observed in the siRNA treatment group $(p<$ 0.05, Fig. 4, A and B). In Fig. 4, C, there was a significant increase $(p<0.05)$ in the TNF- $\alpha$ cytokine secreted in the scr+ACM experimental group compared to the control, however, in the ACM+siRNA group a decreasing trend in TNF- $\alpha$ was observed, but the values are not statistically different. 
The amounts of anti-inflammatory cytokines IL-10 and IL-1RA, in addition to the arginase-1 marker for M2 macrophages are seen in Fig. 4, D, E and F, respectively. It was observed that in the scr+ACM experimental group, there was no significant alteration in the amount of IL-10 secreted compared to the control group, however, there was a significant increase in IL-10 when siRNA was added, compared to the scr+ACM experimental group ( $p<0.05$, Fig. $4, D)$. A significant decrease $(p<0.05)$ of IL-1RA was observed in the scr+ACM experimental group, as compared to the control group, and there was no significant alteration when siRNA was added (Fig. 4, E). There was shown to be no significant alteration in the percentage of cells containing arginase- 1 in the scr+ACM experimental group, however, there was a significant increase in percentage of arginase-1 on M2 macrophages when treated with siRNA compared to both the control group, as well as the scr+ACM group $(p<0.05$, Fig. 4, F). Since arginase-1 is a marker for M2 macrophages, it is indicative that there was an increase post-siRNA treatment. 


\section{Discussion}

Monocytes differentiate into M1 and M2 macrophages. The specific role of each macrophage is paramount for disease progression and/or healing (Fukuda and Aikawa 2013; Singla et al. 2015). M1 macrophages are classically activated in cases of cellular stress, such as infection (Xu et al. 2012). In more chronic inflammatory diseases such as ATH, M1 macrophages, however, can cause more damage to the diseased tissue, as previously discussed (Aoyama et al. 2009; Fukuda and Aikawa 2013). Inhibition of the Notch pathway and increased presence of M2 macrophages has previously shown to assist in healing of diseased tissue (Aoyama et al. 2009; Outtz et al. 2010; Singla et al. 2016). The Notch-1 signaling pathway has also been shown to be an important component for communication during differentiation/polarization; however, further studies to understand the effect of blocking the pathway in primary human monocytes are still lacking. (Fukuda et al. 2012; Outtz et al. 2011). Therefore, in the current study, we examine how human primary monocyte differentiate into M1 and M2 macrophages under stress conditions, and how the inhibition of the Notch-1 pathway affects this differentiation.

DAPT treatment appeared to be successful at inhibition of the Notch -1 pathway. The reduction of M1 macrophages, their associated receptors, and cytokines was observed post-DAPT treatment. Likewise, the M2 population was enhanced after DAPT treatment under stressed cellular conditions.

In a 2010 cancer research study used OP9 cells to study macrophage polarization (Wang et al. 2010). When induced by LPS via the Notch-1 pathway, 
it was found that there was production of pro-inflammatory cytokine IL-12 associated with M1 macrophages, however it was able to be blocked with a GSI, and subsequent increase of M2 macrophages and IL-10 was observed (Wang et al. 2010). This is consistent with our results of the current study, as well as our previous study on THP-1 cells (Singla et al. 2014).

We acknowledge, however, that there is certainly communication between this pathway and other molecular processes in the cell, and further studies needs to be performed. Phenotype switching post treatment is also a possibility in this case, and requires further investigation. Additionally, as mentioned previously, DAPT inhibition is believed to be non-specific, and therefore, in subsequent testing, we used siRNA specific to the Notch-1 receptors.

The data demonstrates that when the primary human monocytes are treated with siRNA, there is significant enhancement of the M2 macrophage population, the presence IL-10, IL-1RA, and the number of cells expressing arginase-1. Concurrently, there was a significant reduction in M1 macrophages as well as percentage of Notch-1 receptors on the macrophages. Furthermore, a significant decrease in IL-6 and MCP-1 was observed when siRNA was added to the ACM media, suggesting that pro-inflammatory cytokine secretion was partially halted by the inhibition of this pathway. Moreover, we found that ACM+siRNA decreases M1 macrophages and increases M2 macrophages. There might be a possibility that siRNA inhibition of the Notch-1 receptor could result in adaptive intracellular responses; therefore, future work is needed to confirm what kind of new compensatory pathways are activated. Additionally, it 
will be interesting to see if treatment with siRNA enhances differentiation of M2 macrophages directly, or if there is M2 macrophage polarization from M1 macrophages generated with ACM treatment.

When comparing the current study versus our previously published THP-1 study by Singla et al., there were similar results when comparing the quantitative data relating to cytokine levels, post- DAPT or siRNA treatment. In our studies, we examined the pro-inflammatory cytokines IL-6, MCP-1 and TNF- $\alpha$, and the anti-inflammatory cytokines IL-10 and IL-1RA.

In our 2014 study on THP-1 cells, it was determined that there was a significant decrease in the pro-inflammatory cytokines IL-6, MCP-1, and TNF- $\alpha$ post-DAPT treatment, as well as post-siRNA treatment compared to the ACM media experimental group ( $p<0.05$, Singla et al. 2014). In our current study post-DAPT treatment, there was a significant decrease $(p<0.05)$ in MCP-1 compared to the ACM experimental group, there was no significant change in IL6 levels, and TNF- $\alpha$ was not examined. Post-siRNA treatment, there was a significant decrease $(p<0.05)$ in the IL-6 and MCP-1 groups compared to the ACM experimental group, and there was no significant change in TNF- $\alpha$ levels.

As for anti-inflammatory cytokines in our 2014 study, there was a significant increase $(p<0.05)$ in both cytokines IL-10 and IL-1RA post DAPT, and post siRNA treatments compared to the ACM experimental group (Singla et al. 2014). In our current study, there was found to be a significant increase in IL10 and IL-1RA $(p<0.05)$ compared to both the control and the experimental ACM group post-DAPT treatment. Additionally, post-siRNA treatment, there was 
e a significant increase $(p<0.05)$ in IL-1RA compared to the ACM experimental group, and no significant change in IL-1RA.

Moreover, another recent study compared how THP-1 cells and primary human monocytes expressed cytokines post-treatment of LPS (Schildberger et al. 2013). When THP-1 cells were treated with LPS, there was secretion of TNFa, similar to that of the primary human monocyte group under the same experimental conditions, however, the THP-1 cells did not secrete IL-6 and IL-10, and secreted IL-8 in a much lower quantities when compared to the primary human monocyte group (Schildberger et al. 2013). This is different than the data presented in the current study when compared to our previous THP-1 study in 2014.

These results obviously differ from that of Schildberger et al., and this raises many questions. The difference in results could be due in part to the Schildberger et al. study using LPS to stimulate the cells, where as we used ACM, DAPT and siRNA to inhibit the functionality of the Notch-1 receptors. The mechanics of their study versus ours are different, and require further investigation. Additionally, it is unknown, as stated previously, if the macrophages switch between M1 and M2 polarization post-treatment, or if they directly differentiate directly to either the M1 or M2 subclass. Furthermore, there was a difference in some of the cytokines examined between studies, which also could be a contributing factor to the difference between our studies and Schildberger et al. 
In an additional recent study by Fukuda et al., it was found that inhibiting the Delta-like ligand 4 (DII-4) improved the effect of the inflammatory process associated with atherosclerosis (Fukuda et al. 2012). It was also found that there was a decrease in MCP-1 levels in the aorta, adipose tissue, and peripheral blood (Fukuda et al. 2012). In the current study, MCP-1 levels were significantly decreased when the Notch-1 pathway was inhibited via DAPT or siRNA. This study corroborates our findings that there is significance in Notch as a key player in inflammatory processes, however, it differs in that it examines a different aspect of the Notch pathway, and also does not examine M2 macrophages, and their receptors and cytokines specifically. Phenotype switching, post-alteration to the Notch pathway, is possible and therefore gives rise to the need for further study of the mechanistic relationships between the Notch pathway and macrophages building on current studies in this area of research.

In summary, our presented data suggests that when the Notch-1 pathway in primary human monocytes is inhibited by either siRNA or DAPT, there is enhancement of M2 macrophage polarization as evidenced by CD206 staining and arginase-1 presence. This is further supported by an increase in the secreted anti-inflammatory IL-10 and II-1RA cytokine levels. Moreover, when we compared the data in the same setting to our previous report on THP-1 cells, primary human monocytes have similar characteristics, at least in the model we generated. Furthermore, DAPT data on these primary human monocytes have very similar effects as observed with Notch-1R specific siRNA. Our data suggest shows that Notch-1 induces M2 macrophage differentiation; however, further 
studies on exact mechanisms and intracellular cell signaling are needed. Moreover, we published BMP-7 as a mediator of macrophage polarization using the SMAD-PI3K-Akt-mTOR pathway in M2 macrophage differentiation (Singla et. al. 2014). It will be interesting to explore whether SMAD-PI3K-Akt-mTOR pathway is a central pathway to enhance M2 macrophage differentiation with Notch-1 activation or whether another pathway regulates M2 macrophage differentiation remains unknown. This study opens a new avenue for future investigations as well as a strong potential to develop novel therapies for inflammatory diseases. 


\section{Acknowledgements}

Authors are thankful to Jessica Hellein for her assistance in drafting the manuscript.

\section{Conflicts of Interest}

The author(s) declare no conflicts of interest. 


\section{References}

Aoyama, T., Takeshita, K., Kikuchi, R., Yamamoto, K., Cheng, X. W., Liao, J. K., and Murohara, T. 2009. [gamma]-Secretase inhibitor reduces diet-induced atherosclerosis in apolipoprotein E-deficient mice. Biochem. Biophys. Res. Commun. 383(2): 216-222.

Daigneault, M., Preston, J. A., Marriott, H. M., Whyte, M. B., and Dockrell, D. H. 2010. The Identification of Markers of Macrophage Differentiation in PMAStimulated THP-1 Cells and Monocyte-Derived Macrophages. PLoS ONE, 5(1): 1-10. doi:10.1371/journal.pone.0008668

Fukuda, D., and Aikawa, M. 2013. Expanding Role of Delta-Like 4 Mediated Notch Signaling in Cardiovascular and Metabolic Diseases. Circ. J. 77(10): 24622468.

Fukuda, D., Aikawa, E., Swirski, F., Novobrantseva, T., Kotelianski, V., Gorgun, C., Chudnovskiy, A., Yamazaki, H., Croce, K., Weissleder, R., Aster, J., Hotamisligil, G., Yagita, H., and Aikawa, M. 2012. Notch ligand Delta-like 4 blockade attenuates atherosclerosis and metabolic disorders. Proc. Natl. Acad. Sci. U.S.A. 109(27): 10763-10764.

Fung, E., Tang, S. M., Canner, J. P., Morishige, K., Arboleda-Velasquez, J. F., Cardoso, A. A., Carlesso, N., Aster, J. C., and Aikawa, M. 2007. Delta-like 4 induces notch signaling in macrophages: implications for inflammation. Circulation, 115(23): 2948-2956.

Kigerl, K. A., Gensel, J. C., Ankeny, D. P., Alexander, J. K., Donnelly, D. J., and Popovich, P. G. 2009. Identification of two distinct macrophage subsets with divergent effects causing either neurotoxicity or regeneration in the injured mouse spinal cord. J. Neurosci. 29(43): 13435-13444. doi:10.1523/JNEUROSCI.3257-09.2009

Kleemann, R., Zadelaar, S., and Kooistra, T. 2008. Cytokines and atherosclerosis: a comprehensive review of studies in mice. Cardiovasc. Res. 79(3): 360-376.

Moore, K., and Tabas, I. 2011. Macrophages in the Pathogenesis of Atherosclerosis. Cell, 145(3): 341-355. doi:10.1016/j.cell.2011.04.005

Moore, K. J., Sheedy, F. J., and Fisher, E. A. 2013. Macrophages in atherosclerosis: a dynamic balance. Nat. Rev. Immunol. 13(10): 709-721. doi:10.1038/nri3520

Outtz, H. H., Wu, J. K., Wang, X., and Kitajewski, J. 2010. Notch1 deficiency results in decreased inflammation during wound healing and regulates vascular 
endothelial growth factor receptor-1 and inflammatory cytokine expression in macrophages. J. Immunol. 185(7): 4363-4373. doi:10.4049/jimmunol.1000720

Outtz, H., Tattersall, I., Kofler, N., Steinbach, N., and Kitajewski, J. 2011. Notch1 controls macrophage recruitment and Notch signaling is activated at sites of endothelial cell anastomosis during retinal angiogenesis in mice. Blood, 118(12): 3436-3439.

Qin, Z. 2012. The use of THP-1 cells as a model for mimicking the function and regulation of monocytes and macrophages in the vasculature. Atherosclerosis, 221(1): 2-11. doi:10.1016/j.atherosclerosis.2011.09.003

Rocher, C., and Singla, D. K. 2013. SMAD-PI3K-Akt-mTOR Pathway Mediates BMP-7 Polarization of Monocytes into M2 Macrophages. PLoS ONE, 8(12): 1-9. doi:10.1371/journal.pone.0084009

Rocher, C., Singla, R., Singal, P. K., Parthasarathy, S., and Singla, D. K. 2012. Bone morphogenetic protein 7 polarizes THP-1 cells into M2 macrophages. Can. J. Physiol. Pharmacol. 90(7): 947-951.

Schildberger, A., Rossmanith, E., Eichhorn, T., Strassl, K., and Weber, V. 2013. Monocytes, Peripheral Blood Mononuclear Cells, and THP-1 Cells Exhibit Different Cytokine Expression Patterns following Stimulation with Lipopolysaccharide. Mediat. Inflamm. 2013: Article ID 697972: 1-10. doi:10.1155/2013/697972

Singla, R., Wang, J., and Singla, D. 2014. Regulation of Notch 1 signaling in THP-1 cells enhances M-2 macrophage differentiation. Am. J. Physiol. Heart Circ. Physiol. 307(11): H1634-H1642.

Singla, D. K., Singla, R. D., Abdelli, L. S., and Glass, C. 2015. Fibroblast Growth Factor-9 Enhances M2 Macrophage Differentiation and Attenuates Adverse Cardiac Remodeling in the Infarcted Diabetic Heart. PLoS ONE, 10(3): 1-13. doi:10.1371/journal.pone.0120739

Singla, D. K., Singla, R., and Wang, J. 2016. BMP-7 Treatment Increases M2 Macrophage Differentiation and Reduces Inflammation and Plaque Formation in Apo E-/- Mice. PLoS ONE, 11(1): 1-17. doi:10.1371/journal.pone.0147897

Wang, Y., He, F., Feng, F., Liu, X., Dong, G., Qin, H., Hu, X., Zheng, M., Liang, L., Feng, L., Liang, Y., and Han, H. 2010. Notch Signaling Determines the M1 versus M2 Polarization of Macrophages in Antitumor Immune Responses. Cancer Res. 70(12): 4840-4849. 
Wolfs, I., Donners, M., and de Winther, M. 2011. Differentiation factors and cytokines in the atherosclerotic plaque micro-environment as a trigger for macrophage polarisation. Thromb. Haemostasis. 106(5): 763-771.

Xu, H., Zhu, J., Smith, S., Foldi, J., Zhao, B., Chung, A., Outtz, H., Kitajewski, J., Shi, C., Weber, S., Saftig, P., Li, Y., Ozato, K., Blobel, C., Ivashkiv, L., and Hu, X. 2012. Notch-RBP-J signaling regulates the transcription factor IRF8 to promote inflammatory macrophage polarization. Nature Immunol. 13(7): 642-650. doi:10.1038/ni.2304 


\section{Figures Legends}

Figure: 1. Effect of DAPT on Notch-1 signaling pathway, and subsequent effects on polarized macrophage subclasses. A is composed of photomicrographs for M1 macrophage control and treatment groups, and B is composed of photomicrographs for M2 macrophage control and treatment groups. The images above show the cells are stained with anti-Notch1 $(a, f, k)$ in both $A$ and $B$, antiinducible nitric oxide synthase (iNOS, M1 macrophages) (b, g, l) in A, anti-CD206 (M2 macrophages) (b, g, I) in B, 4',6-diamidino-2-phenylindole (DAPI) (c, h, m) in $A$ and $B$, the merged photomicrographs $(d, i, n)$ in $A$ and $B$, and enhanced versions of the merged images for better visualization $(e, j, o)$ in $A$ and $B$. The scale is $50 \mu \mathrm{m}$. Histograms representing the percentage of $\mathrm{M} 1$ and $\mathrm{M} 2$ macrophages containing the Notch-1 receptor are shown in $\mathrm{C}$ and $\mathrm{E}$, respectively, and the yields of each polarized macrophage are seen in $D$ and $F$, respectively. C-HM cells, control human primary monocytic cells; ACM, human primary monocytic cells cultured in apoptotic-conditioned media; ACM+DAPT, human primary monocytic cells and DAPT cultured in apoptotic-conditioned media. ${ }^{*} p<0.05$ compared to C-HM cells, $\# p<0.05$ compared to ACM. $n=4$ for all groups

Figure: 2. Treatment of human primary monocytes with DAPT and its effect on inflammatory cytokines. Histograms A and B show the quantity of proinflammatory cytokines secreted (IL-6 and MCP-1, respectively). Histograms C and $D$ show the quantity of anti-inflammatory cytokines secreted (IL-10, and IL- 
1RA, respectively), and histogram $E$ depicts the percentage of cells containing the anti-inflammatory marker arginase-1. Quantities obtained by ELISA analysis.

Figure. 3. Effects of siRNA treatment on primary human monocytes and subsequent effects on polarized $M 1(A)$ and $M 2(B)$ macrophages. $A$ is composed of photomicrographs for M1 macrophage control and treatment groups, and B is composed of photomicrographs for M2 macrophage control and treatment groups. See Figure 1 for staining explanation. The scale is $50 \mu \mathrm{m}$. Histograms representing the percentage of $\mathrm{M} 1$ and $\mathrm{M} 2$ macrophages containing the Notch-1 receptor are shown in $\mathrm{C}$ and $\mathrm{E}$, respectively, and the yields of each polarized macrophage are seen in $D$ and $F$, respectively. Con+scr, control human primary monocytic cells and scramble RNA; scr+ACM, human primary monocytic cells and scramble RNA cultured in apoptotic-conditioned media; siRNA+ACM, human primary monocytic cells and siRNA cultured in apoptoticconditioned media. ${ }^{*} p<0.05$ compared to scr-HM cells, \#p<0.05 compared to scr+ACM. $n=4$ for all groups

Figure 4: Treatment of human primary monocytes with siRNA and its effect on inflammatory cytokines. Histograms $A, B$, and $C$ show the quantity of proinflammatory cytokines secreted (IL-6, MCP-1, and TNF- $\alpha$, respectively). Bargraphs $\mathrm{D}$ and $\mathrm{E}$ show the quantity of anti-inflammatory cytokines secreted (IL10 and IL-1RA, respectively). Quantities were obtained by ELISA analysis. Bar graph $\mathrm{F}$ depicts the percentage of cells containing the M2 macrophage marker 
arginase-1. A.U., arbitrary units. ${ }^{*} p<0.05$ compared to scr-HM cells, $\# p<0.05$ compared to scr+ACM. $n=4$ for all groups, A.U., arbitrary units. ${ }^{*} p<0.05$ compared to C-HM cells, $\# p<0.05$ compared to ACM. $n=4$ for all groups 
Singla et al. Figure 1
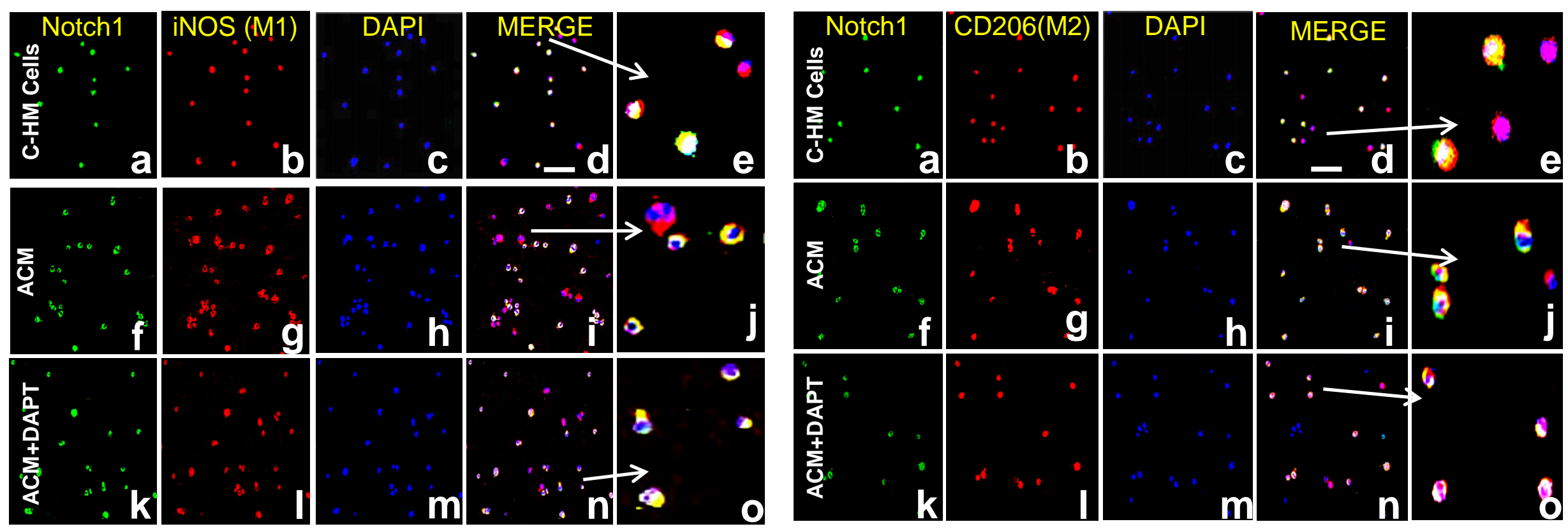

C

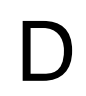

E
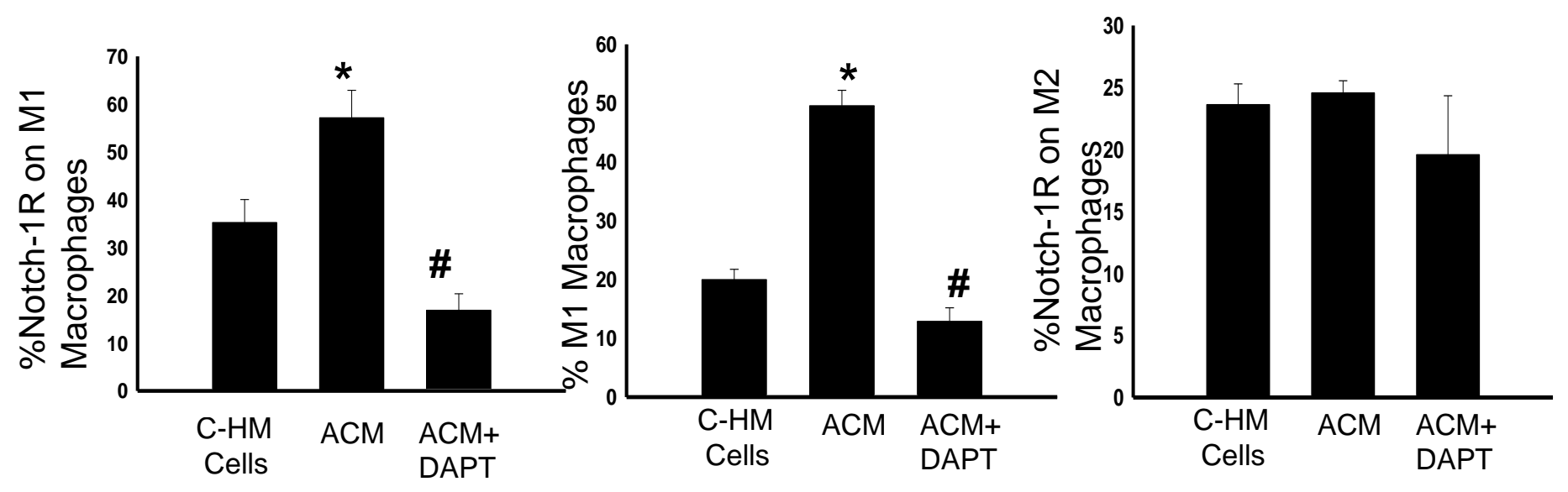

$\mathrm{F}^{\text {Scale bar }=50 u m}$

https://mc06.manuscriptcentral.com/cjpp-pubs

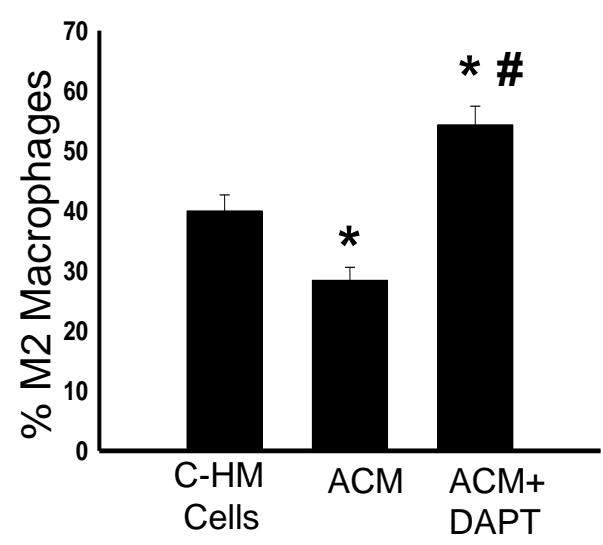


A
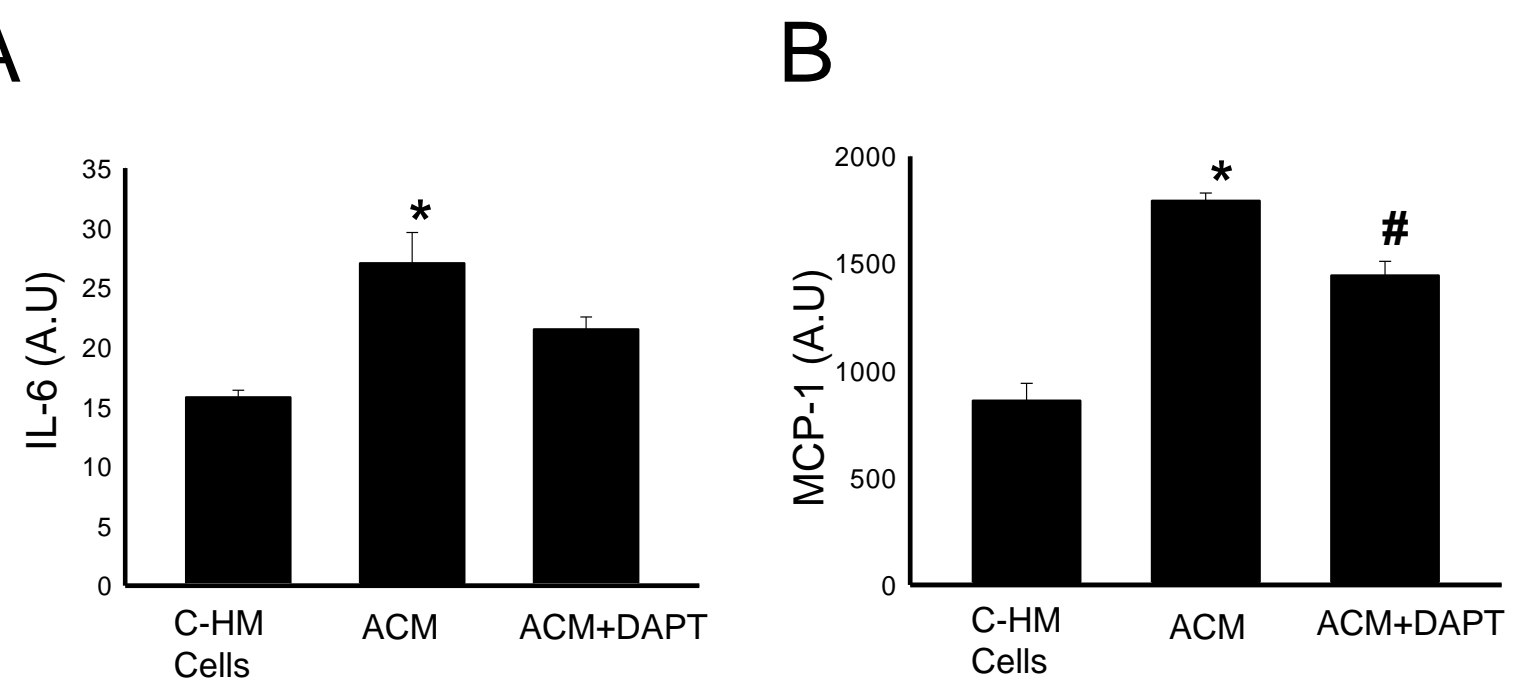

C
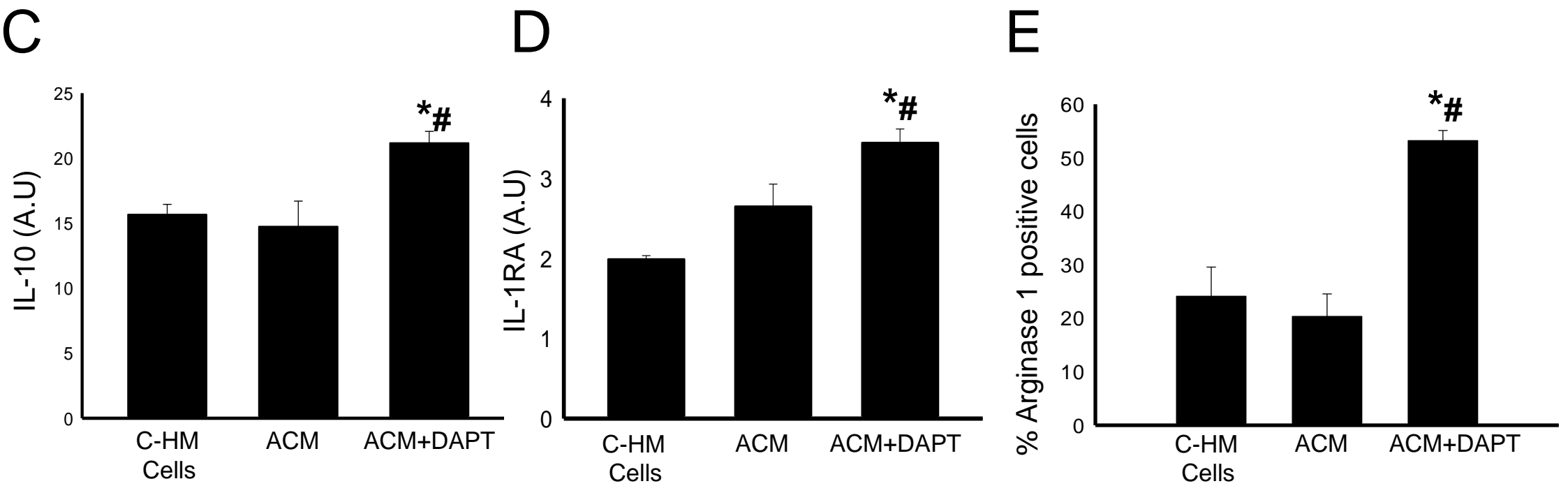

https://mc06.manuscriptcentral.com/cjpp-pubs 

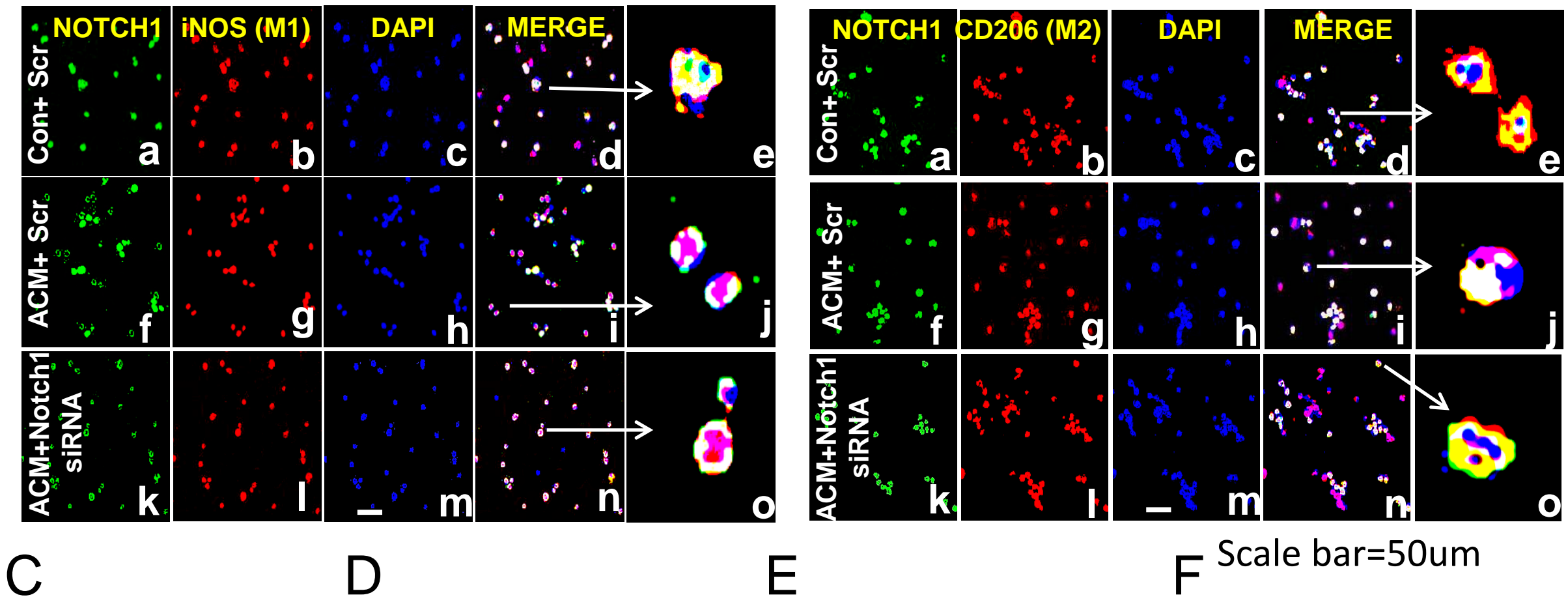

F Scale bar=50um
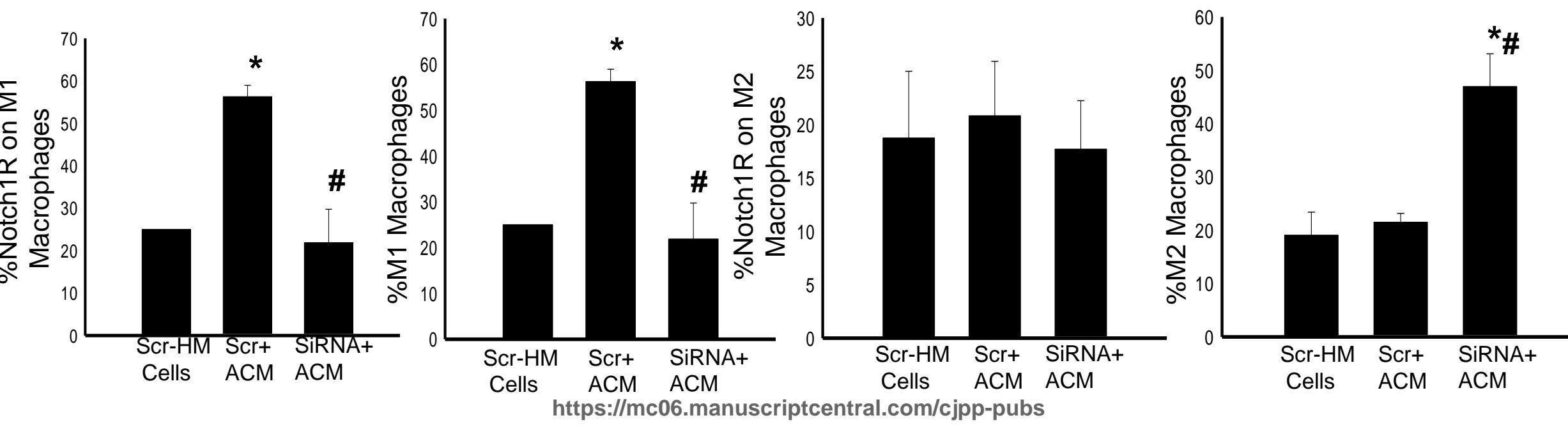


\section{Canadian Journal of Physiology and Pharmacology}

A

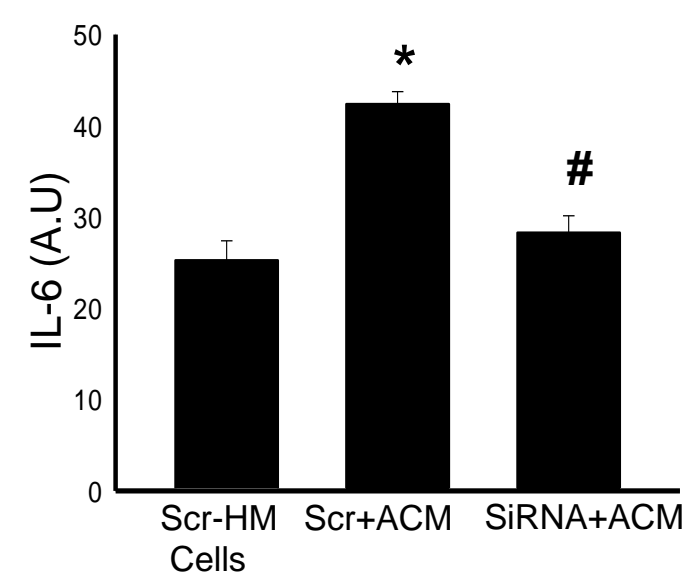

D

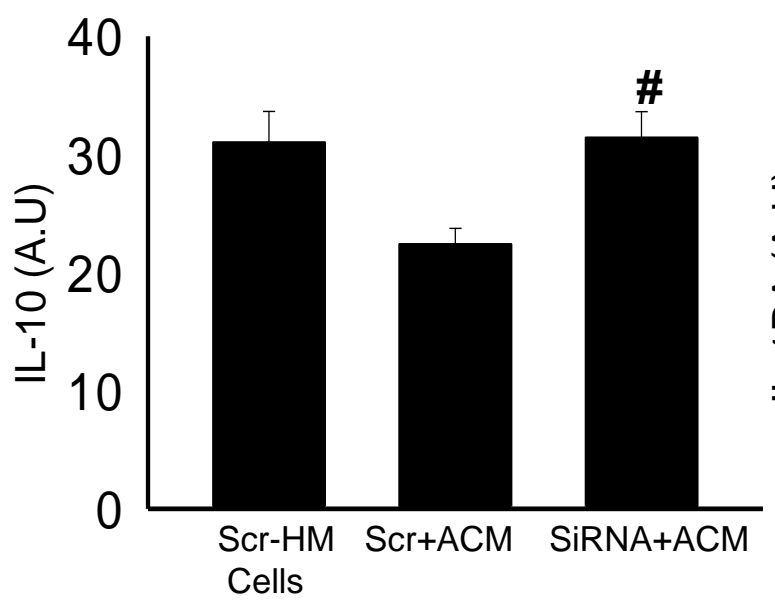

$\mathrm{B}$

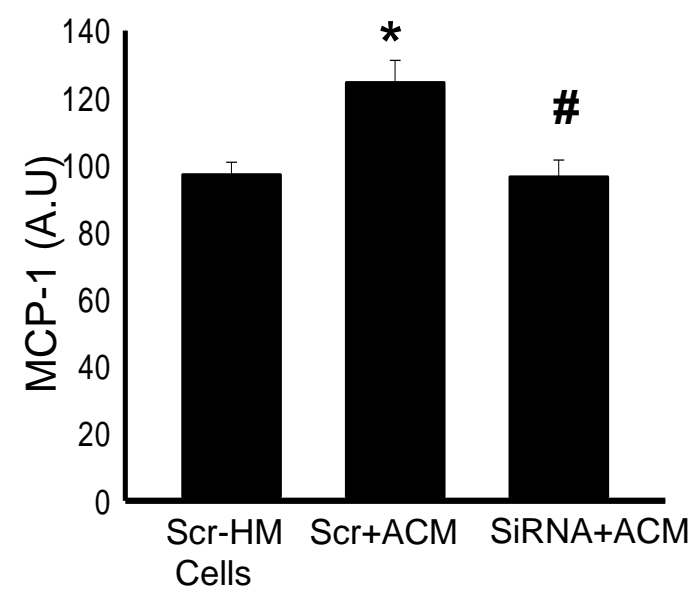

$E$

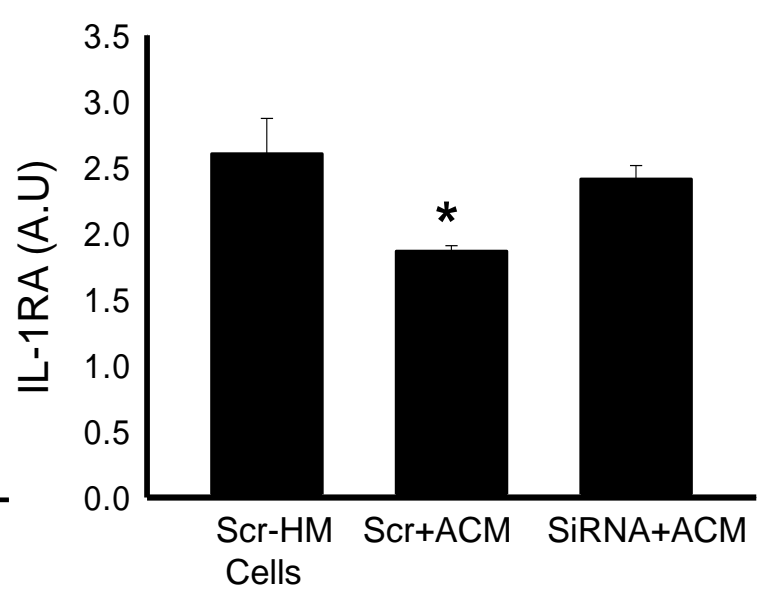

C

Singla et al. Figgdøe $\$ 30$

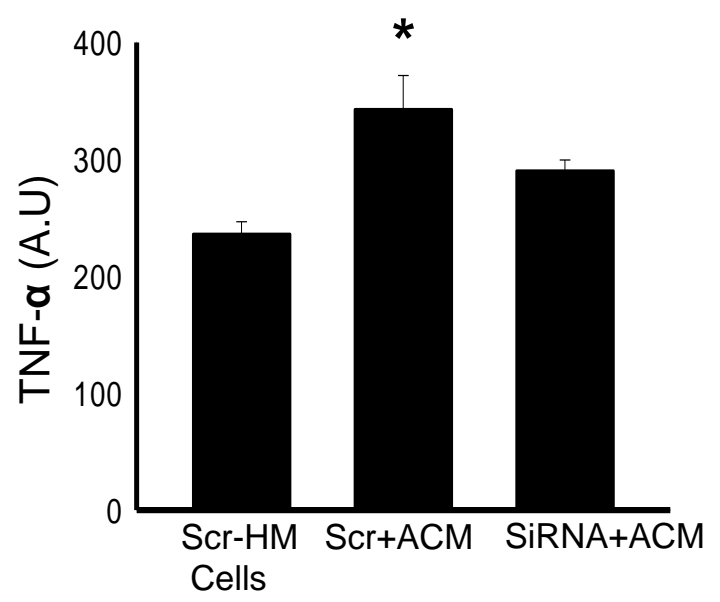

$\mathrm{F}$

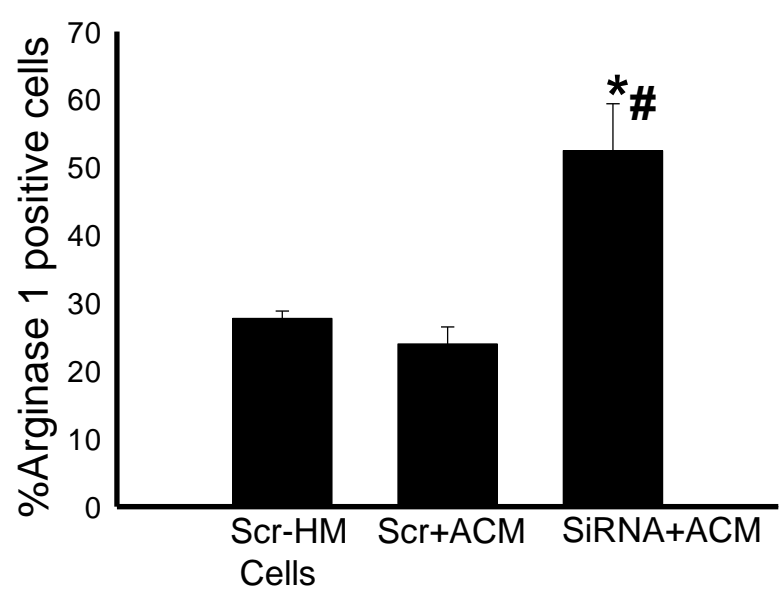

